\title{
Automobile Authentication and Tracking System
}

\author{
N.Duraichi ${ }^{\mathrm{a}, 1}$, K.Arun Kumar ${ }^{\mathrm{b}}$, N.Lokesh Sathya ${ }^{\mathrm{b}}$, S.Lokesh ${ }^{\mathrm{b}}$ \\ aProfessor, Department of Electronics and Communication Engineering, \\ ${ }^{b} U G$ Student, Department of Electronics and Communication Engineering, \\ Vel Tech High Tech Dr Rangarajan Dr Sakunthala Engineering College, Chennai, TN, \\ India
}

\begin{abstract}
Vehicle robbery and unknown car thefts has become a intense issue around the nation. Many culprits use unapproved vehicles to perform numerous illegal activities and leave the vehicles. The utmost reason for accidents is due to the vehicles driven by unknown users, who perform reckless and inexperienced driving without the speed limit will cause many accidents that increases the death rate. Our goal is to make a system which will allow the person who have authorized license. For this purpose, we plan to install an automated system in the vehicle to introduce smart license verification technology. Various techniques and technologies are being explained to detect the details of the driver, and also Various vehicle thefts are being done in spite of various surveillance cameras are set down to keep an eye on the activities and various technologies are being implemented to diminish the vehicle robbery. So, we proposed the system with the concept of deep learning. As compared to normal detection techniques deep learning collects $\mathrm{N}$ number of input samples and compares it with the database details. After the authentication process the engine mechanism starts, if not authorized it gives a buzzer sound and vehicle doesn't start until the details of registered person is authenticated.
\end{abstract}

Keywords. Deep Learning Techniques, Image Processing, Arduino ATmega328 microcontroller

\section{Introduction}

Nowadays, we can see that many people who doesn't have license (children below age 18) are driving vehicles without thinking about the accident or the danger it can create for them and as well as others [1]. Our aim is to make a system that which allows only the person who has the authorized driving license. For this purpose, we need to set up a system in vehicle. For input we use the RFID tags and for output digital screen will be used [2]. Now the person who wants to drive has to place his RFID tag near the Reader and then the system will verify it with the central data. If the input details are wrong then the system will not start and the person cannot drive the vehicle. If the detail in the RFID tag matches with the details in the central data, then the vehicle will start and the person can drive the vehicle now [3].

\footnotetext{
${ }^{1}$ Dr.N.Duraichi, Department of Electronics and Communication Engineering, Vel Tech High Tech Dr Rangarajan Dr Sakunthala Engineering College, TN, India; Email: duraichi@velhightech.com.
} 


\section{Existing System}

In the existing system, there were no advanced techniques used. The only way to verify the drivers was to stop the vehicle in the traffic and verify the driver's license and this causes the traffic jam in the peak hours. The checking police or RTO'S cannot check each and every vehicle in the traffic section and the rate of accident have not been reduced [4].

\section{Disadvantages:}

- Manual method is used and rate of accident is high

- Time consumption for verification is high.

\section{Proposed System}

As shown in Figure1 and Figure 2, In our project we are using the Infrared Sensor which will detect the whether the person is sitting in the driver seat and the details will be sent the microcontroller. If the Infrared Red sensor detects the person, then he has inserted the key. The user has to place the RFID tag in the reader and in that the driver's license details is fed in the micro controller and it reads the details of the driver [5]. From the microcontroller the instructions will be sent to the camera and it will take the picture of the driver and then the captured picture will be compared with the picture stored in the system. If both the details of the person match with the stored details then the car mechanism will turn ON the car and if the details doesn't match then the buzzer which will give the alarm. By this setup we can check the driver license Addition to this setup we are adding checking device in which we need setup a checking lane at the tollgates and check posts to receive the details of the driver using the ZIGBEE module and it will be uploaded in the server through the IOT module [6]. And if the Infrared sensor didn't sense the presence of person, then the details will be sent to the microcontroller and from there the car will be immediately turned off with the help of motor mechanism. From that we are able to trace the car theft and the driver without license which will reduce the rate of accidents [7].

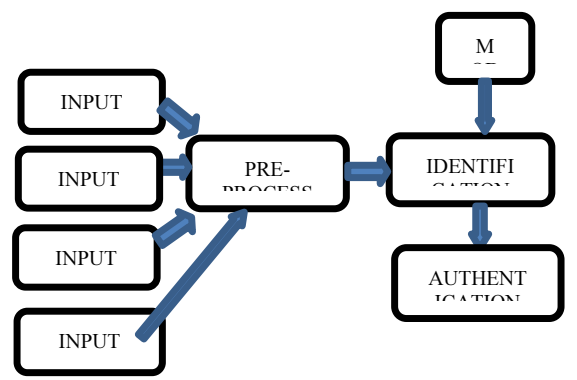

Figure 1. Block Diagram for Testing Section

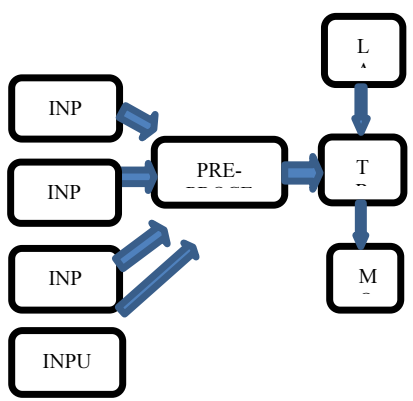

Figure 2. Block Diagram for Training Section

\subsection{Operating principle}

When looking for somebody, the machine will take a photo of them to check their identity. This is referred to as a 1:1 verification process; if we search for a specific person's photo in a database, it is referred to as a 1: $\mathrm{N}$ search. In both cases, the device 
will generate a corresponding remuneration result, either success or annoyance. The block diagram depicts details about face enrolment testing and preparation [8]. After attaching the camera to the PC, we must first open the computer and repeat the enrolment of the same person image several times. Once the face enrolment is complete, we can add the approved license ID number and the authorized person's Aadhar card number, and then that information will be saved in Centralized database [9].

\subsection{ARDUINO ATmega328}

Arduino is a software and hardware platform for projects and users that designs and produces microcontroller kits for creating digital devices and interactive objects that sense and manage objects in both the physical and digital worlds. A variety of microprocessors and controllers are used on the Arduino board. The boards have digital and analogue input/output (I/O) pins that can be connected to a variety of expansion boards (shields) or breadboards (other circuits on them). Arduino's architecture, or more precisely, Arduino's integrated circuits (ATmega328p). The ATmega328/P is a low-power CMOS 8-bit microcontroller with an AVR instruction set that reduces risk. The AVR uses Harvard design to optimize efficiency and similarity, with separate reminiscences and buses for program and details. With one stage of pipelining, instruction inside the program memory region is dead. Associate degree external $16 \mathrm{MHz}$ oscillator controls the clock [10].

\subsection{USB Camera}

The USB camera is a gadget that is used to capture a picture of the person. Image of a person will give us a scope for the quick identification of a particular person. Face capturing system are widely used for security purpose due to its acceptability, accuracy, Feasibility, and reliability.

\subsection{Image Processing}

Image processing functions are based on uniformity measurements are used to obtain better performance person authentication, creating an image of the particular person. The image processing technique is used to extract high resolution images from the lowresolution images by removing the noise in it. A CNN application, perfectly trained, provides suitable for particular person face recognition when used in coexisting with a previously developed face recognition module. It is also shown that image processing functions based on SIFT measurements can be used to obtain performance object features location, creating a key point face reorganization for every feature. A grid of Scale Invariant Feature Transform (SIFT) key points was drawn and a neural network was used also for vehicle model recognition.

\subsection{Deep Learning}

Deep learning is a machine learning subcategory that employs multiple layers to derive progressively such higher-level options from raw inputs. Lower layers in an image processing technique, for example, could decide edges, while higher layers could 
determine concepts related to human identification, such as numbers, letters, ears, or fingerprints. Every stage learns to rework its input file into a variety of abstract, linear, and composite representations using this technique. In a picture recognition program, the raw input could be a matrix of pixels; the primary realistic layer could abstract the pixels and write edges; and the secondary realistic layer could abstract the pixels and write edges. The second layer could write and compose edge arrangements; the third layer could write a nose and eyes, and thus the fourth layer could recognize that the image contained a face. Significantly, a deep learning system can learn on its own which options to best position within which stage.

\section{4. $\quad$ Results}

Here in the proposed system, we will place an IR Sensor, when the IR Sensor detects the person, it will display the "P DETECT" on the screen as shown in the Figure 3.

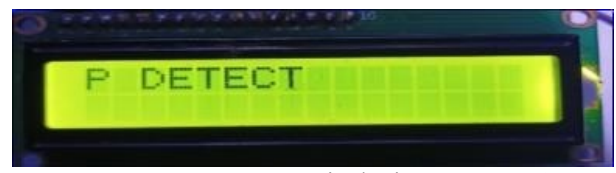

Figure 3. Displaying P DETECT

Next after the person detected by the IR Sensor, we need to place the RFID Tag near the RFID Reader. Here in my proposed model, we assigned the name of the RFID Tag as ARUN. So, it displays "P DETECTARUN" on the Screen as shown in the Figure 4.

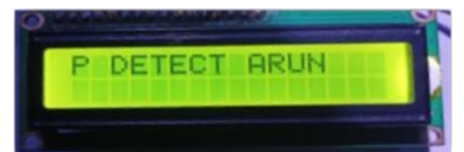

Figure 4. Displaying P DETECT ARUN

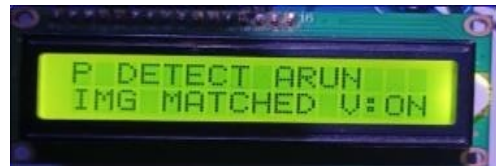

Figure 5. Displays IMG MATCHED V:ON

Once after the RFID Tag reading all the details will be stored in the device will be checked with the driver sitting in the driver seat. Then it displays "IMG MATCHED $\mathrm{V}: \mathrm{ON}$ " on the screen as shown in the Figure 5 and Figure 6 and the vehicles will starts. Whenever an unauthorized person tries to drive the vehicle the buzzer goes $\mathrm{ON}$ and it will displays the "IMG NOT MATCV:OF" on the screen as shown Figure 7, Figure 8 and Figure 9.

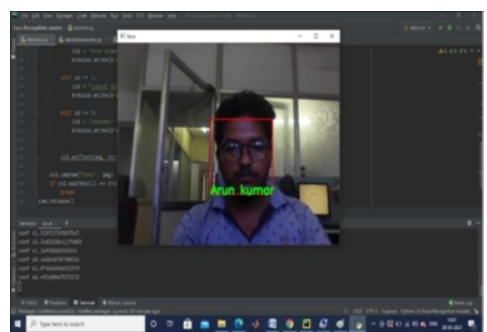

Figure 6. Image Verification Process

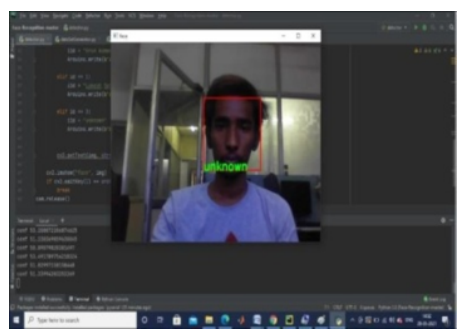

Figure 7. Displays IMG NOT MATCHED V:OFF 


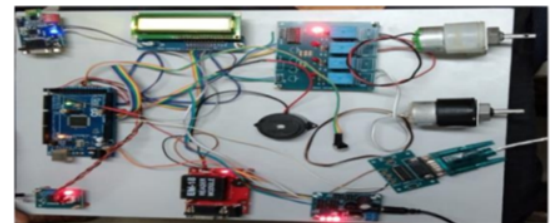

Figure 8. Experimental Setup of the Car Section

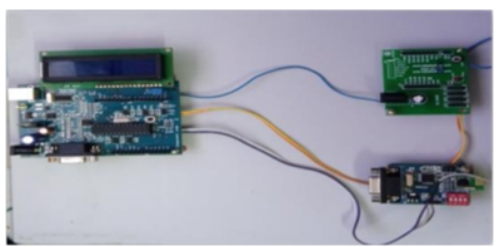

Figure 9. Experimental Setup of the Toll Gate Section

\section{Conclusion}

Proposed method is sketched for verifying the driving license basing on Arduino ATmega328 and face authentication are designed to achieve the gadgets by coding which are essential for the face authentication. This system integrates both the intersection and coding using PyCharm and Arduino ATmega328 microcontroller. This system can be future enhanced by using different techniques like Deep Neural Network (DNN) etc. This system can be utilized for the authentication of the driving license, detection of fake license for decreasing the crime and accidents rate.

\section{References}

[1] Deep Das, P., \& Sengupta, S. (2017). "Implementing the next-generation system to protect vehicles from thefts and accidents". 2017 International Conference on Innovations in Green Energy and Healthcare Technologies(IGEHT).doi:10.1109/igeht.2017.8094045

[2] Rajalakshmi, G. (2015). Real time smart car lock security system using face detection and recognition. International Journal of Applied Engineering Research, 10(4), 11307-11312

[3] Luo, D., Lu, X., Hu, C., Peng, X., \& Wang, W. (2017). A robust traffic driver face detection method. In PIC 2016 - Proceedings of the 2016 IEEE International Conference on Progress in Informatics and Computing (pp. 285-288). Institute of Electrical and Electronics Engineers Inc. https://doi.org/10.1109/PIC.2016.7949511

[4] Saifullah, Khawaja, A., Hamza, Arsalan, Maryam, \& Anum. (2010). Keyless car entry through face recognition using FPGA. In 2010 International Conference on Future Information Technology and Management Engineering, FITME 2010 (Vol. 1, pp. 224-227). https://doi.org/10.1109/FITME.2010.5654862

[5] Kolli, A., Fasih, A., Al Machot, F., \& Kyamakya, K. (2011). Non-intrusive car driver's emotion recognition using thermal camera. In Proceedings of the Joint 3rd International Workshop on Nonlinear Dynamics and Synchronization, INDS'11 and 16th International Symposium on Theoretical Electrical Engineering, ISTET'11 (pp. 156-160). https://doi.org/10.1109/INDS.2011.6024802

[6] Faisal, M., \& Thakur, A. (2016). Autonomous car system using facial recognition and geo location services. In Proceedings of the 2016 6th International Conference - Cloud System and Big Data Engineering, Confluence 2016 (pp. 417-420). Institute of Electrical and Electronics Engineers Inc. https://doi.org/10.1109/CONFLUENCE.2016.7508155

[7] Zhixiong, L. (2005). A new embedded car theft detection system. In ICESS 2005 - Second International Conference on Embedded Software and Systems (Vol. 2005, pp. 89-94). https://doi.org/10.1109/ICESS.2005.6

[8] Al-Shemarry, M. S., Li, Y., \& Abdulla, S. (2020). An Efficient Texture Descriptor for the Detection of License Plates from Vehicle Images in Difficult Conditions. IEEE Transactions on Intelligent Transportation Systems, 21(2), 553-564. https://doi.org/10.1109/TITS.2019.2897990

[9] Du, S., Ibrahim, M., Shehata, M., \& Badawy, W. (2013). Automatic license plate recognition (ALPR): A state-of-the-art review. IEEE Transactions on Circuits and Systems for Video Technology. https://doi.org/10.1109/TCSVT.2012.2203741

[10] Huang, R., Tawfik, H., \& Nagar, A. K. (2009). Licence plate character recognition based on support vector machines with clonal selection and fish swarm algorithms. In 11th International Conference on Computer Modelling and Simulation, UKSim 2009 (pp. 101-106). https://doi.org/10.1109/UKSIM.2009.64 